\title{
Magnitude and trend of perinatal mortality and its relationship with inter-pregnancy interval in Ethiopia: a systematic review and meta-analysis
}

Belayneh Hamdela Jena ${ }^{1 *}$ (D), Gashaw Andargie Biks², Kassahun Alemu Gelaye ${ }^{3}$ and Yigzaw Kebede Gete ${ }^{3}$

\begin{abstract}
Background: Perinatal mortality remains a problem in Ethiopia. Findings of primary studies varied on level of perinatal mortality and its predictors including inter-pregnancy interval. The aim of this review was to estimate the pooled perinatal mortality rate, its trend overtime and verify the association with inter-pregnancy interval in Ethiopian context.

Methods: Studies were accessed through the electronic web-based search strategies from PubMed, ScienceDirect, Hinari for health via Research4Life, Google and Advanced Google search, and retrieving via relevant references using a combination of medical subject headings (MeSH terms) and key words related with inter-pregnancy interval. $R$ version 3.4.3 software was used for the meta-analysis. A forest plot and $\mathrm{I}^{2}$ test were done to assess heterogeneity. Sensitivity analysis and subgroup analysis were done to deal with heterogeneity. A weighted inverse variance random-effects model was applied to estimate pooled effect sizes. A funnel plot and Egger's regression test were done to check publication bias.

Results: A total of 34 studies used to answer review questions (30 for perinatal mortality rate and its trend estimation from 1997 to 2019 and 8 for its relationship with inter-pregnancy interval). The pooled perinatal mortality rate was 51.3 per 1000 total births (95\% Cl: 40.8-62.8). The pooled stillbirth rate was 36.9 per 1000 births (95\% Cl: 27.3-47.8) and early neonatal mortality rate was 29.5 per 1000 live births (95\% Cl: 23.9-35.6). Increasing trend was seen in stillbirth rate (23.7 to 36.9 per 1000 births) while decreasing trend in early neonatal mortality rate (51 to 29.5 per 1000 live births). Slight reduction trend was observed in overall perinatal mortality rate (66 to 51.3 per 1000 births). An inter-pregnancy interval less than 15 months was found to be statistically significantly associated with perinatal mortality; pooled $\mathrm{OR}=2.76$ (95\% Cl: 2.1-3.62). Spacing pregnancy for at least 15 months was related with reducing perinatal mortality by $64 \%$ (95\% Cl: $52.38,72.38 \%)$.
\end{abstract}

Conclusions: In Ethiopia, perinatal mortality rate remains high. Insignificant reduction trend was observed in overall perinatal mortality rate. Counseling couples about the importance of spacing pregnancy and intensifying longacting contraceptive use will help in reducing perinatal mortality related to poor pregnancy spacing.

Keywords: Inter-pregnancy interval, Perinatal mortality, Stillbirth, Early neonatal death, Ethiopia

\footnotetext{
* Correspondence: bhamdela@gmail.com

'Department of Public Health, College of Medicine and Health Sciences, Wachemo University, Hossana, Ethiopia

Full list of author information is available at the end of the article
}

(c) The Author(s). 2020 Open Access This article is licensed under a Creative Commons Attribution 4.0 International License, which permits use, sharing, adaptation, distribution and reproduction in any medium or format, as long as you give appropriate credit to the original author(s) and the source, provide a link to the Creative Commons licence, and indicate if changes were made. The images or other third party material in this article are included in the article's Creative Commons licence, unless indicated otherwise in a credit line to the material. If material is not included in the article's Creative Commons licence and your intended use is not permitted by statutory regulation or exceeds the permitted use, you will need to obtain permission directly from the copyright holder. To view a copy of this licence, visit http://creativecommons.org/licenses/by/4.0/. The Creative Commons Public Domain Dedication waiver (http://creativecommons.org/publicdomain/zero/1.0/) applies to the data made available in this article, unless otherwise stated in a credit line to the data. 


\section{Background}

Perinatal mortality is defined as fetal loss at or after 28 weeks of gestation (stillbirth) or neonatal death within 7 days of life (early neonatal mortality) [1].

Globally, of the estimated 3 million perinatal deaths that occur in each year, low and middle income countries share the highest burden (97-99\%). Perinatal mortality is a reflection of poor socio-economic status of a country, poor maternal health service utilization and the quality of obstetric and neonatal care facilities available [2-4]. Furthermore, inappropriate maternal health care provision during the course of pregnancy, labor, delivery and postpartum periods, particularly when complications happen and lack of newborn care immediately after delivery and within the first 7 days of life were the main contributing factors for the highest burden of perinatal death in low resource settings [4].

In Ethiopia, perinatal mortality is one of the highest in Africa, 46 per 1000 pregnancies [2]. It is mainly attributed to home delivery, which accounts for more than $75 \%$ of the perinatal deaths [5]. Reducing neonatal, infant and under five mortalities were a global agenda. Ethiopia shared sustainable development goal (SDG) to achieve the target for reduction of neonatal mortality to below 12 per 1000 live births, by 2030 [6]. Reduction of neonatal, infant and under-five mortalities may not be realized without substantial reduction of perinatal mortality. This is because of the fact that most of the neonatal deaths occur during the first week of life, which is a part of perinatal mortality [4, 7, 8]. Thus, all interventions aimed at reducing neonatal, infant and underfive mortalities are expected to begin with intervention during the perinatal periods. Of course, the Ministry Of Health, Ethiopia, had been working for years to make health services accessible for women through community and facility based interventions to increase newborn and child survival (Unpublished). Despite these interventions, perinatal mortality remains one of the problems in Ethiopia, particularly; home delivery remains the challenge to reduce perinatal mortality. Still, most (74\%) women give birth outside health institution without skilled care attendant [9]. Studies indicated that the causes of stillbirth and early neonatal death are closely related [10] so that the risk factors for both of them can be studied together as perinatal mortality. With regard to this, studies identified multiple risk factors for perinatal mortality such as prematurity, low birth weight, previous history of perinatal death, not receiving tetanus toxoid immunization, lack of Iron supplementation etc. $[1,3,11,12]$. On the other side, closely spaced pregnancies are hypothesized as one of the risk factors for poor perinatal outcomes [13]. Few studies conducted in Ethiopia attempted to see the relationship between inter-pregnancy interval and perinatal mortality. However, there were inconsistencies among these studies. Some studies reported that there was relationship $[1,3]$ while others reported no relationship $[11,12$, 14]. To come up with evidence based interventions, clarifying the relationship is vital. In this regard, the researchers' hypothesis was that; short inter-pregnancy interval of less than 15 months would have increased the risk of perinatal mortality as compared to interpregnancy interval of greater than or equal to 15 months in Ethiopia.

It is obvious that systematic review and metaanalysis yield higher level of evidence for policy and decision making. Evidence on the pooled effect sizes for overall perinatal mortality, stillbirth and early neonatal mortality was limited in Ethiopia. Cumulative evidence (trend) of perinatal mortality, stillbirth and early neonatal mortality were inadequate to see the progresses over time and give subsequent attention. More than half of Ethiopian women have the short interval between pregnancies [2]. Further attention is needed to increase inter-pregnancy interval. Thus, ascertaining its relationship with perinatal mortality is crucial.

Therefore, this systematic review and meta-analysis was aimed to address the following review questions:

1. What is the pooled estimate of a perinatal mortality rate in Ethiopia?

2. What was the trend of perinatal mortality over time in Ethiopia?

3. Was inter-pregnancy interval less than 15 months associated with perinatal mortality as compared to its counterpart in Ethiopia?

\section{Methods}

\section{Reporting and protocol registration}

The results of this systematic review and meta-analysis were reported based on the Preferred Reporting Items for Systematic Review and Meta-Analysis statement (PRISMA 2009) guideline with 27 items checklist [15] (See Additional file 1). The protocol was registered in the PROSPERO Database: (PROSPERO 2019: CRD42019125186) Available from: https://www.crd.york.ac.uk/PROSPERO.

\section{Inclusion criteria}

Observational study designs (cross-sectional, case referent and cohort/follow up studies) were included in the review. Studies that observed the proportion of at least one of the perinatal outcomes (stillbirth and/or early neonatal death) and/or the relationship between short inter-pregnancy interval or birth interval and any one of the perinatal outcomes were considered. We considered articles published in English language, 
have relevant full text and from Ethiopia. There was no time restriction for the articles published in order to see the trend over time.

\section{Exclusion criteria}

Studies that considered only high risk mother for perinatal death such as studies done on mothers with pregnancy related hypertensive disorders, ante-partum hemorrhage, obstructed labor etc. were excluded as these conditions have known effect on perinatal outcomes. Hence, it might exaggerate the effect size and the pooled estimates might be misleading. Qualitative studies were also excluded since it is not appropriate to quantify effect sizes. Finally, we did not consider interventional studies in this review.

\section{Information sources and search strategies}

Databases (PubMed, ScienceDirect, and Hinari for health via Research4Life) and grey literatures sources (Google, Advanced Google Scholar) were accessed. Further search was made through snowballing or retrieving from relevant references used in related studies. A combination of medical subject headings (MeSH terms) using Boolean operators and key words related with inter-pregnancy interval were used to search studies.

The last date to access databases was made in December, 3/2019 on Tuesday until 2:50 PM. [See Additional file 2].

\section{Study selection}

Retrieved studies were exported to reference manager software, Endnote version 7. Duplicated studies were removed using the Endnote and manually. Two independent reviewers (Belayneh Hamdela (BH) and Alemu Ersido (AE)) screened the title and abstract for the relevance. During this preliminary assessment, reading title and abstract, primary studies found to be irrelevant were excluded. Two reviewers $(\mathrm{BH}$ and AE) participated in study selection by reading titles and abstracts only. When disagreement between two reviewers happened, the third reviewer (Solomon Hailemeskel $(\mathrm{SH})$ ) was used to handle the disagreement based on the relevance for pre-specified objectives and inclusion criteria. Then primary studies with only relevant information and fulfills inclusion criteria were selected for full text review and reason for exclusion were presented using PRISMA flow diagram.

\section{Risk of Bias assessment}

Two independent reviewers (BH and $\mathrm{AE}$ ) appraised the quality of each study. The Joanna Briggs Institute (JBI) quality appraisal checklist was used for each study design [16]. The disagreement between two reviewers ( $\mathrm{BH}$ and $\mathrm{AE}$ ) was resolved by involving third reviewer $(\mathrm{SH})$. Cohen's Kappa statistics was used to calculate degree of agreement between the two reviewers ( $\mathrm{BH}$ and $\mathrm{AE})$.

For each of the three study designs, a study was considered low risk of bias score of $50 \%$ and above of the quality assessment indicators. Studies with low risk of bias were included for the meta-analysis.

\section{Data extraction process}

Data extraction form was prepared and piloted using excel spread sheet. Two forms were prepared: one for the magnitude and trend of perinatal mortality (including stillbirth and early neonatal mortality), and the other form was for the risk factor of perinatal mortality (i.e. inter-pregnancy interval). The form prepared for perinatal mortality contained: study id, region, study setting, authors and year of publication, study design, sample size and proportion of perinatal mortality. As most women did not remember last normal menstrual period, inter-pregnancy interval (IPI) was usually estimated from the birth interval by subtracting 9 months of gestation [2]. In this review, the main exposure variable was inter-pregnancy interval less than 15 months or birth interval less than 24 months. With this regard, the other form, in addition to those mentioned above, contains data for $2 \times 2$ table: perinatal death among exposed (IPI $<15$ months), survived among exposed, perinatal death among unexposed (IPI $>=15$ months), survived among unexposed, exposed total, unexposed total and the Odds Ratios (ORs). Two reviewers (BH and $\mathrm{AE}$ ) extracted the data on the forms and cross checked for any disagreements. The extracted data then edited and saved in a comma delimited (CSV) file format to suit for the analysis.

\section{Data synthesis and analysis}

The data extracted and saved in CSV format in excel spread sheet was imported to $\mathrm{R}$ version 3.4.3 statistical software for the analysis. Before pooling the effect sizes, the summary measures were transformed to suitable transformations that result in normal distribution. Accordingly, the proportion of perinatal mortality was transformed to Freeman and Tukey double arcsine transformation (PFT). Double arcsine transformation was recommended for proportions when the observed proportions of each study are either below 0.20 or above 0.80. In such a cases log transformation might have a limitation of stabilizing variance. Therefore, Freeman and Tukey double arcsine transformation was recommended to solve the limitation of log transformation or to stabilize the variance so that it gives more valid estimate of the weighted average. On the other hand, the 
odds ratios of the individual studies were transformed in to its $\log$ scale. All analyses were done by using transformed summary measures. Back translation of summary measures was done for reporting as this help readers easily understand the results. Heterogeneity among studies was checked by two methods. Firstly, subjective method (forest plot) was used to visualize graphically. Secondly, more objectively tested and quantified using the I-squared statistic, in which 25,50 , and $75 \%$ represented low, moderate and high heterogeneity respectively [17]. Random effects model was used to estimate the pooled effect size using Dar-Simonial Liard (DL). Tau squared was used to quantify the amount of heterogeneity in random effect model. To deal with heterogeneity, sensitivity and subgroup analyses were done. Sub group analysis was done by using year of publication, study settings, regions and designs. To report the pooled effect sizes from the included studies, meta-analysis of pooled proportion and odds ratio were done. Potential publication bias was checked using funnel plot and Egger's regression test. Overall trends of perinatal mortality rates were examined both graphically and using simple linear regression test. Data for trend analysis is available from 1997 to 2019 years. Since there was no data for all years we grouped cumulative trends for every 5 years to suit for better interpretation of trend line. $P$-value less than 0.05 was used to describe statistically significant decreasing trend in perinatal mortality rate.

\section{Results}

\section{Selection of studies}

A total of 918 studies were retrieved from sources by pre-specified search strategies. Of these, 47 studies were duplicates and are removed. In preliminary screening, 815 studies ( 802 by looking at the title and 13 by abstract) were removed. A total of 56 studies were identified for checking eligibility for full text review. Of these, 21 studies were excluded with the following reasons:

A case control study from Bonga General and Mizan Tepi University Teaching Hospitals, a facility based case control study from Arbaminch general hospital, a prospective cohort study from tertiary care hospitals in Addis Ababa, a case control study from public hospitals of Tigray, a prospective cohort study from Northern Ethiopia, a cross-sectional study on neonatal mortality from Ethiopia, a case control study from Debre Tabor town, a hospital based retrospective cohort study from Somali Ethiopia, a case control study from Hawassa University hospital were excluded due to lack of relevant data for this review. A 5 years retrospective survey from Tikur Anbesa Hospital, a matched case control study from Kalu district, a retrospective chart review from Yekatit 12 hospital, Addis Ababa, a cross-sectional study from Addis Ababa hospital, a cross-sectional study from Jimma Hospital and a retrospective review on perinatal mortality audit from Jimma hospital were excluded due to lack of access to full text. A retrospective study from Mettu Karl referral hospital Ethiopia, a cross-sectional study from Amhara region hospitals, an institutional based cross-sectional study from Debremarkos referral hospital and a retrospective cohort study from Wolita Sodo referral hospital were excluded since they were conducted on high risk groups for perinatal deaths. A study from Kersa demographic and health surveillance system site was excluded due to double reporting in publications. Lastly, an interventional study from Ethiopia was excluded because of the design as this review focus on observational studies.

Finally, a total of 35 studies that fulfill the prespecified inclusion criteria were obtained (Fig. 1).

\section{Characteristics of included studies}

A total of 35 studies included in this review. However, studies included in the meta-analysis were thirty four $[1-3,8,11,12,18-45]$. Pregnant women, women attending for labor and delivery service and newborns were study participants considered for data source in primary studies.

Of 34 studies included in the meta-analysis, 30 studies contained data relevant for perinatal mortality and trend estimation. From 30 studies, 25(83.3\%) and 18(60\%) studies had data relevant for pooling stillbirth and early neonatal mortality rates, respectively. One study (25) had crudely reported perinatal mortality without specifying either stillbirth or early neonatal mortality. From 34 studies, only $8(23.5 \%)$ contained data relevant to see the relationship between inter-pregnancy interval and perinatal mortality.

Perinatal mortality rate (PMR) in primary studies ranges from 13.98 (15) to 85.3 (24) per 1000 total births in community based studies. Whilst it ranges from 27.6 (25) to 173 (23) per 1000 total births in health institution based studies. Stillbirth rate (SBR) ranges from 5.9 (26) to 85.3 (24) per 1000 total births in community based studies while from 22.7 (31) to 173.3(23) per 1000 total births at health institution based studies. Early neonatal mortality rate (ENMR) ranges from 10.8(26) to 41.4(32) per 1000 live births in community based studies while it ranges from $24.8(5)$ to $110(30)$ per 1000 live births at health institution. All conditions (PMR, SBR and ENMR) were higher in health facility based studies than community based studies. Inter-pregnancy interval less than 15 months or birth interval less than 24 months was significantly associated with perinatal mortality in $5(62.5 \%)$ of the 8 studies. In two studies however (20, 


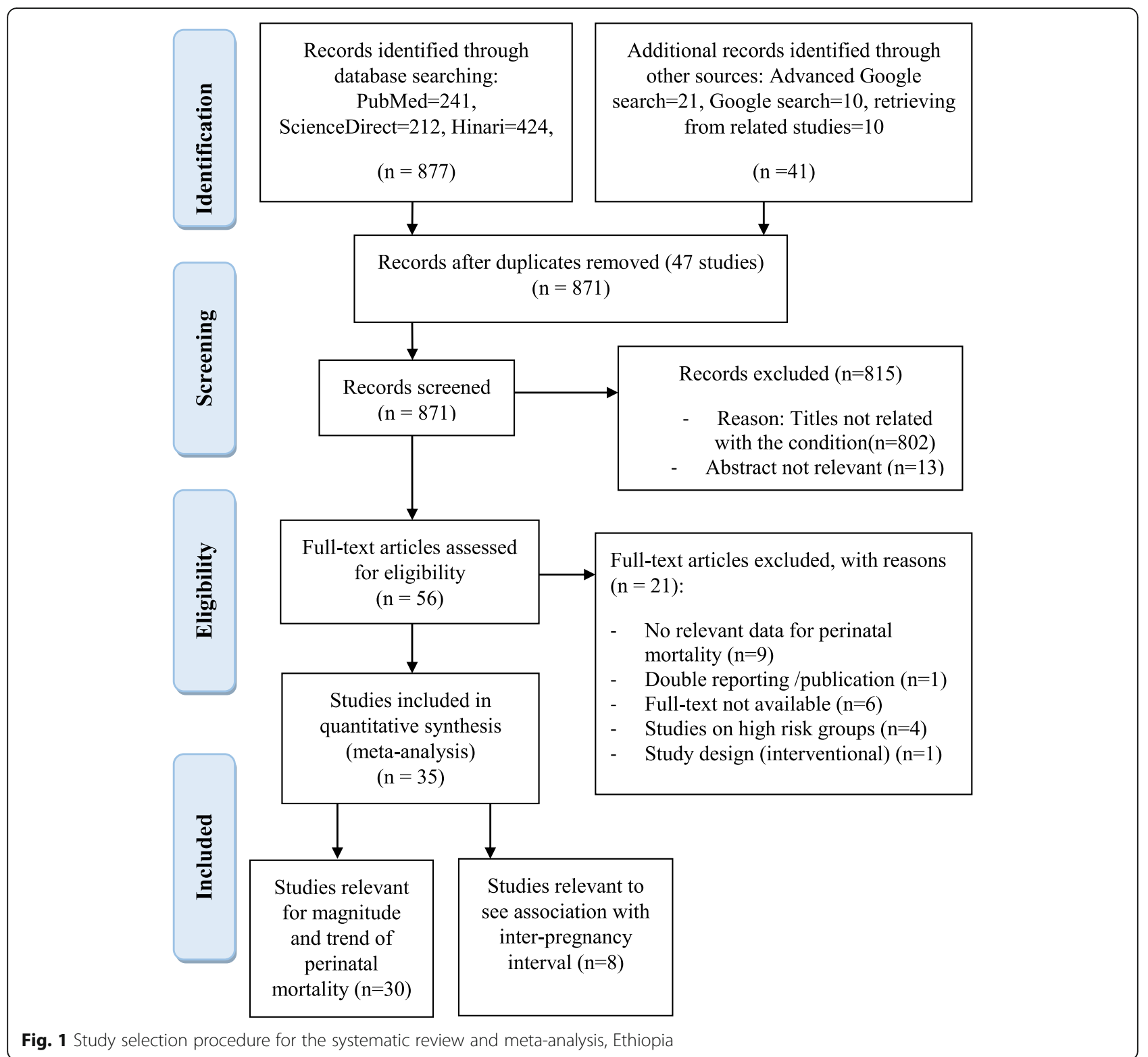

26) it was not significantly associated with perinatal mortality. Categorization of inter-pregnancy interval or birth interval in these two studies was not in line with most of the literatures. In one study (34), it is unclear that, whether it was fitted in the adjusted model or not.

Of 34 studies, $26(76.4 \%)$ were conducted in both (urban and rural) settings. The others: $4(11.8 \%)$ in urban and $4(11.8 \%)$ in rural settings alone. Sixteen (47.1\%) were community based while $18(52.9 \%)$ were institution based studies. Regarding regions: Amhara 8(23.6\%), Oromia 6(17.7\%), SNNP 5(14.7\%), Addis Ababa 3(8.8\%), Tigray 5(14.7\%), Benishangul-Gumuz 1(2.9\%), mixed (more than one region) $3(8.8 \%$ ) and the others 3(8.8\%) were National (Ethiopia) Demographic and Health
Surveys (EDHS). Concerning the study design: crosssectional $21(61.8 \%)$, follow ups $6(17.6 \%)$, prospective cohort $1(2.9 \%)$, case control $4(11.8 \%)$, nested case control 2(5.9\%).

Regarding year of publication: 2(5.9\%) published before 2000, 11(32.3\%) published from 2001 to 2015 and 21(61.8\%) published from 2016 to 2019.

Study id, author, year of publication, region, study design, sample size, exposure variable and outcome data were extracted to describe the characteristics of the studies. The total sample size of the studies was 141,835 $(136,168$ to determine the magnitude of perinatal mortality and 5667 to see the relationship between inter-pregnancy interval and perinatal mortality). A 
Table 1 Characteristics of primary studies included in the systematic review and meta-analysis, 2019, Ethiopia

\begin{tabular}{|c|c|c|c|c|c|c|c|c|c|c|c|c|}
\hline $\begin{array}{l}\text { Study } \\
\text { ID }\end{array}$ & Author (year) & Region & $\begin{array}{l}\text { Study } \\
\text { Design }\end{array}$ & $\begin{array}{l}\text { Sample } \\
\text { Size }\end{array}$ & $\begin{array}{l}\text { PMR per } \\
1000 \\
\text { total } \\
\text { births }\end{array}$ & $\begin{array}{l}\text { SBR } \\
\text { per } \\
1000 \\
\text { births }\end{array}$ & $\begin{array}{l}\text { ENM R } \\
\text { per } 1000 \\
\text { live births }\end{array}$ & $\begin{array}{l}\mathrm{IPI}<15 \\
\text { months } \\
\text { with PM }\end{array}$ & $\begin{array}{l}\mathrm{IPI}<15 \\
\text { months } \\
\text { without } \\
\text { PM }\end{array}$ & $\begin{array}{l}|P| \geq 15 \\
\text { months } \\
\text { with PM }\end{array}$ & $\begin{array}{l}\mathrm{IPI} \geq 15 \\
\text { months } \\
\text { without } \\
\mathrm{PM}\end{array}$ & $\begin{array}{l}\text { OR } \\
{[95 \% \mathrm{Cl}]}\end{array}$ \\
\hline 1 & $\begin{array}{l}\text { Sahlemariam Y (1997) } \\
\text { [36] }\end{array}$ & Addis Ababa & Follow up & 1606 & $\begin{array}{l}106 / 1606 \\
(66)\end{array}$ & $\begin{array}{l}38 / 1606 \\
(23.7)\end{array}$ & $\begin{array}{l}68 / 1334 \\
\mathbf{( 5 0 . 9 )}\end{array}$ & - & - & - & - & - \\
\hline 2 & EDHS (2000) [32] & National & Cross sectional & 12,494 & $\begin{array}{l}655 / 12494 \\
\mathbf{( 5 2 )}\end{array}$ & $\begin{array}{l}\text { 234/12494 } \\
\mathbf{( 1 8 . 7 )}\end{array}$ & $\begin{array}{l}421 / \\
12260 \\
\mathbf{( 3 4 )}\end{array}$ & - & - & - & - & - \\
\hline 3 & EDHS (2005) [33] & National & Cross sectional & 11,280 & $\begin{array}{l}420 / 11280 \\
\text { (37) }\end{array}$ & $\begin{array}{l}117 / 11280 \\
(\mathbf{1 0 . 4 )}\end{array}$ & $\begin{array}{l}303 / \\
10860 \\
\mathbf{( 2 7 . 9 )}\end{array}$ & - & - & - & - & - \\
\hline 4 & EDHS (2011) [2] & National & Cross sectional & 12,077 & $\begin{array}{l}551 / 12077 \\
\mathbf{( 4 6 )}\end{array}$ & $\begin{array}{l}\text { 204/12077 } \\
\mathbf{( 1 6 . 9 )}\end{array}$ & $\begin{array}{l}347 / \\
11873 \\
\mathbf{( 2 9 )}\end{array}$ & - & - & - & - & - \\
\hline 5 & Chekol A (2011) [26] & Benishangul & Cross sectional & 581 & $\begin{array}{l}69 / 581 \\
\mathbf{( 1 1 8 . 8 )}\end{array}$ & $\begin{array}{l}56 / 581 \\
\mathbf{( 9 6 . 4 )}\end{array}$ & $\begin{array}{l}13 / 525 \\
\mathbf{( 2 4 . 8 )}\end{array}$ & - & - & - & - & - \\
\hline 6 & Assefa N (2012) [22] & Oromia & $\begin{array}{l}\text { prospective } \\
\text { follow up }\end{array}$ & 1438 & $\begin{array}{l}27 / 1438 \\
\mathbf{( 1 8 . 8 )}\end{array}$ & $\begin{array}{l}27 / 1438 \\
\mathbf{( 1 8 . 8 )}\end{array}$ & - & - & - & - & - & - \\
\hline 7 & Wakgari N (2013) [41] & mixed (EDHS) & Cross sectional & 8651 & $\begin{array}{l}343 / 8651 \\
(\mathbf{3 9 . 6 )}\end{array}$ & - & $\begin{array}{l}343 / 8651 \\
(39.6)\end{array}$ & - & - & - & - & - \\
\hline 8 & Andargie G (2013) [3] & Amhara & $\begin{array}{l}\text { Prospective } \\
\text { longitudinal }\end{array}$ & 1752 & $\begin{array}{l}88 / 1752 \\
(\mathbf{5 0 . 2 )}\end{array}$ & $\begin{array}{l}41 / 1752 \\
(23.4)\end{array}$ & $\begin{array}{l}47 / 1711 \\
(27.5)\end{array}$ & 40 & 483 & 48 & 1881 & $\begin{array}{l}2.04[1.32 ; \\
3.14]\end{array}$ \\
\hline 9 & Worku A (2014) [44] & Amhara & $\begin{array}{l}\text { Prospective } \\
\text { Cohort }\end{array}$ & 727 & $\begin{array}{l}36 / 727 \\
(\mathbf{4 9 . 5 2 )}\end{array}$ & $\begin{array}{l}\text { 20/727 } \\
\mathbf{( 2 7 . 5 )}\end{array}$ & $\begin{array}{l}16 / 707 \\
(22.6)\end{array}$ & - & - & - & - & - \\
\hline 10 & Adane A (2014) [19] & Amhara & Cross sectional & 481 & $\begin{array}{l}34 / 481 \\
(70.7)\end{array}$ & $\begin{array}{l}34 / 481 \\
(70.7)\end{array}$ & - & - & - & - & - & - \\
\hline 11 & Yaya Y (2014) [45] & SNNP & Cross sectional & 11,762 & $\begin{array}{l}\text { 226/11762 } \\
\mathbf{( 1 9 . 2 )}\end{array}$ & $\begin{array}{l}226 / 11762 \\
(\mathbf{1 9 . 2 )}\end{array}$ & - & - & - & - & - & - \\
\hline 12 & $\begin{array}{l}\text { Debelew G (2014) } \\
\text { [28] }\end{array}$ & Oromia & $\begin{array}{l}\text { Prospective } \\
\text { follow up }\end{array}$ & 3510 & $\begin{array}{l}123 / 3510 \\
\text { (35) }\end{array}$ & $\begin{array}{l}47 / 3510 \\
(\mathbf{1 3 . 4 )}\end{array}$ & $\begin{array}{l}76 / 3463 \\
(\mathbf{2 1 . 9 )}\end{array}$ & - & - & - & - & - \\
\hline 13 & Abdo R (2016) [18] & SNNP & Cross sectional & 327 & $\begin{array}{l}28 / 327 \\
\mathbf{( 8 5 . 6 )}\end{array}$ & $\begin{array}{l}28 / 327 \\
\mathbf{( 8 5 . 6 )}\end{array}$ & - & - & - & - & - & - \\
\hline 14 & $\begin{array}{l}\text { Mengesha H (2016) } \\
\text { [34] }\end{array}$ & Tigray & $\begin{array}{l}\text { prospective } \\
\text { cohort }\end{array}$ & 1152 & $\begin{array}{l}50 / 1152 \\
(\mathbf{4 3 . 4 )}\end{array}$ & - & $\begin{array}{l}50 / 1152 \\
(\mathbf{4 3 . 4 )}\end{array}$ & - & - & - & - & - \\
\hline 15 & Shifa G (2016) [8] & SNNP & Cross sectional & 20,161 & $\begin{array}{l}282 / 20161 \\
(\mathbf{1 3 . 9 8 )}\end{array}$ & - & $\begin{array}{l}282 / \\
20161 \\
\mathbf{( 1 3 . 9 8 )}\end{array}$ & - & - & - & - & - \\
\hline 16 & Berhie K (2016) [25] & mixed & Cross sectional & 12,560 & $\begin{array}{l}320 / 12560 \\
(\mathbf{2 5 . 5 )}\end{array}$ & - & $\begin{array}{l}320 / \\
12560 \\
\mathbf{( 2 5 . 5 )}\end{array}$ & - & - & - & - & - \\
\hline 17 & Asefa D (2016) [21] & Oromia & Cross sectional & 9921 & $\begin{array}{l}\text { 1080/9921 } \\
(\mathbf{1 0 8 . 9 )}\end{array}$ & $\begin{array}{l}852 / 9921 \\
\mathbf{( 8 5 . 9 )}\end{array}$ & $\begin{array}{l}232 / 9069 \\
(\mathbf{2 5 . 6 )}\end{array}$ & - & - & - & - & - \\
\hline 18 & Aragaw Y (2016) [20] & Oromia & Cross sectional & 3786 & $\begin{array}{l}372 / 3786 \\
\mathbf{( 9 8 . 3 )}\end{array}$ & $\begin{array}{l}\text { 268/3786 } \\
(\mathbf{7 0 . 8 )}\end{array}$ & $\begin{array}{l}104 / 3518 \\
(29.6)\end{array}$ & - & - & - & - & - \\
\hline 19 & Ballard K (2016) [23] & $\begin{array}{l}\text { mixed (Amhara } \\
\text { and Oromia) }\end{array}$ & Cross sectional & 4358 & $\begin{array}{l}95 / 4358 \\
(21.8)\end{array}$ & $\begin{array}{l}95 / 4358 \\
(21.8)\end{array}$ & - & - & - & - & - & - \\
\hline 20 & Yirgu R (2016) [12] & Amhara & $\begin{array}{l}\text { Nested Case } \\
\text { control }\end{array}$ & 4049 & $\begin{array}{l}102 / 4049 \\
\mathbf{( 2 5 . 2 )}\end{array}$ & $\begin{array}{l}57 / 4049 \\
(\mathbf{1 4 . 1 )}\end{array}$ & $\begin{array}{l}45 / 3992 \\
(\mathbf{1 1 . 3 )}\end{array}$ & 1 & 5 & 101 & 217 & $\begin{array}{l}0.43[0.05 ; \\
3.73]\end{array}$ \\
\hline 21 & Cherie N (2017) [27] & Amhara & Cross sectional & 462 & $\begin{array}{l}21 / 462 \\
(\mathbf{4 5 . 5 )}\end{array}$ & $\begin{array}{l}21 / 462 \\
(\mathbf{4 5 . 5 )}\end{array}$ & - & - & - & - & - & - \\
\hline 22 & Dejene T (2017) [38] & Oromia & Cross sectional & 413 & $\begin{array}{l}33 / 413 \\
(\mathbf{7 9 . 9 )}\end{array}$ & $\begin{array}{l}33 / 413 \\
(\mathbf{7 9 . 9 )}\end{array}$ & - & - & - & - & - & - \\
\hline 23 & Mihiretu A (2017) [35] & SNNP & Cross sectional & 300 & $\begin{array}{l}52 / 300 \\
(173.3)\end{array}$ & $\begin{array}{l}52 / 300 \\
(173.3)\end{array}$ & - & - & - & - & - & - \\
\hline 24 & Lakew D (2017) [31] & Amhara & Cross sectional & 2555 & $\begin{array}{l}218 / 2555 \\
\mathbf{( 8 5 . 3 )}\end{array}$ & $\begin{array}{l}218 / 2555 \\
\mathbf{( 8 5 . 3 )}\end{array}$ & - & 104 & 793 & 43 & 1009 & $\begin{array}{l}3.08[2.13 ; \\
4.44]\end{array}$ \\
\hline 25 & Tsegaye B (2018) [40] & SNNP & Cross sectional & 580 & $\begin{array}{l}16 / 580 \\
\mathbf{( 2 7 . 6 )}\end{array}$ & - & - & - & - & - & - & - \\
\hline 26 & Roro E (2018) [11] & Oromia & $\begin{array}{l}\text { Nested Case } \\
\text { control }\end{array}$ & 4383 & $\begin{array}{l}73 / 4383 \\
(16.7)\end{array}$ & $\begin{array}{l}26 / 4383 \\
\mathbf{( 5 . 9 )}\end{array}$ & $\begin{array}{l}47 / 4357 \\
(\mathbf{1 0 . 8 )}\end{array}$ & 15 & 18 & 58 & 128 & $\begin{array}{l}1.84[0.87 ; \\
3.9]\end{array}$ \\
\hline 27 & Goba G (2018) [1] & Tigray & unmatched case & 265 & - & - & - & 42 & 39 & 49 & 135 & $2.97[1.72 ;$ \\
\hline
\end{tabular}


Table 1 Characteristics of primary studies included in the systematic review and meta-analysis, 2019, Ethiopia (Continued)

\begin{tabular}{|c|c|c|c|c|c|c|c|c|c|c|c|c|}
\hline $\begin{array}{l}\text { Study } \\
\text { ID }\end{array}$ & Author (year) & Region & $\begin{array}{l}\text { Study } \\
\text { Design }\end{array}$ & $\begin{array}{l}\text { Sample } \\
\text { Size }\end{array}$ & $\begin{array}{l}\text { PMR per } \\
1000 \\
\text { total } \\
\text { births }\end{array}$ & $\begin{array}{l}\text { SBR } \\
\text { per } \\
1000 \\
\text { births }\end{array}$ & $\begin{array}{l}\text { ENM R } \\
\text { per } 1000 \\
\text { live births }\end{array}$ & $\begin{array}{l}\mid \mathrm{PI}<15 \\
\text { months } \\
\text { with PM }\end{array}$ & $\begin{array}{l}\mathrm{IPI}<15 \\
\text { months } \\
\text { without } \\
\text { PM }\end{array}$ & $\begin{array}{l}|P| \geq 15 \\
\text { months } \\
\text { with PM }\end{array}$ & $\begin{array}{l}\mid \mathrm{PI} \geq 15 \\
\text { months } \\
\text { without } \\
\mathrm{PM}\end{array}$ & $\begin{array}{l}\text { OR } \\
{[95 \% \mathrm{Cl}]}\end{array}$ \\
\hline & & & control & & & & & & & & & $5.12]$ \\
\hline 28 & Getiye Y (2017) [29] & Addis Ababa & $\begin{array}{l}\text { unmatched case } \\
\text { control }\end{array}$ & 395 & - & - & - & 41 & 29 & 69 & 256 & $\begin{array}{l}5.25[3.04 ; \\
9.04]\end{array}$ \\
\hline 29 & Tilahun S (2008) [39] & Addis Ababa & $\begin{array}{l}\text { unmatched case } \\
\text { control }\end{array}$ & 343 & - & - & - & 27 & 27 & 93 & 196 & $\begin{array}{l}2.11[1.17 ; \\
3.79]\end{array}$ \\
\hline 30 & Tewabe T (2018) [37] & Amhara & Cross sectional & 391 & $\begin{array}{l}43 / 391 \\
(\mathbf{1 0 9 . 9 )}\end{array}$ & & $\begin{array}{l}43 / 391 \\
(\mathbf{1 0 9 . 9 )}\end{array}$ & - & - & - & - & - \\
\hline 31 & Haftu A (2018) [30] & Tigray & $\begin{array}{l}\text { prospective } \\
\text { cohort }\end{array}$ & 1103 & $\begin{array}{l}55 / 1103 \\
\mathbf{( 4 9 . 9 )}\end{array}$ & $\begin{array}{l}25 / 1103 \\
\mathbf{( 2 2 . 7 )}\end{array}$ & $\begin{array}{l}30 / 1078 \\
(\mathbf{2 7 . 8 )}\end{array}$ & - & - & - & - & - \\
\hline 32 & $\begin{array}{l}\text { Woldeamanuel B } \\
\text { (2019) [42] }\end{array}$ & Mixed (EDHS) & Cross sectional & 2738 & $\begin{array}{l}170 / 2738 \\
(\mathbf{6 2 . 0 9 )}\end{array}$ & $\begin{array}{l}59 / 2738 \\
(\mathbf{2 1 . 5 5 )}\end{array}$ & $\begin{array}{l}111 / 2679 \\
\mathbf{( 4 1 . 4 3 )}\end{array}$ & - & - & - & - & - \\
\hline 33 & Berhe T (2019) [24] & Tigray & cross sectional & 570 & $\begin{array}{l}21 / 570 \\
\mathbf{( 3 6 . 8 )}\end{array}$ & $\begin{array}{l}21 / 570 \\
\mathbf{( 3 6 . 8 )}\end{array}$ & & - & - & - & - & - \\
\hline 34 & Worede D (2019) [43] & Amhara & $\begin{array}{l}\text { unmatched case } \\
\text { control }\end{array}$ & 420 & & & & 49 & 98 & 35 & 238 & $\begin{array}{l}3.4[2.08 ; \\
5.57]\end{array}$ \\
\hline
\end{tabular}

National: Study conducted at national level (Ethiopia) like DHS

Mixed: when study considered data for more than one region in Ethiopia

sample size considered for primary studies ranges from 300 to 20,161. For the assessment of the relationship between inter-pregnancy interval and perinatal mortality, the sample size considered ranges from, 219 to 1949 (See Table 1).

\section{Quality (risk of bias) assessment for the included studies}

Of 24 cross-sectional studies: except for 4 studies (ID: 2, $24,15,33)$, measurement of exposure in a valid and reliable way was unclear for $16(66.6 \%)$ studies. The confounder factors identified way was unclear for 9 studies (ID: 21, 5, 22, 11, 17, 18, 23, 19, 16). Strategies to deal with confounding factors were also unclear for 2 studies (ID: 17, 19). None of the case control studies used matching for potential confounders. From the follow up studies; strategies to address the incomplete follow up was not utilized in 4 studies (ID: 1, 6, 9, 14). According to Cohen's Kappa statistics $(\mathrm{K}=0.65, \%$ of agreement $=$ 97.2\%), the two reviewers (BH and $\mathrm{AE}$ ) have "substantial agreement" to include studies in the meta-analysis [46]. One study [47] was not considered for data extraction since it scores below $50 \%$ for JBI quality indicators. See Table S1 in Additional file 3 for quality assessment scores.

\section{Pooled estimate of perinatal mortality rate}

Forest plot was used to identify heterogeneity between studies. The result of the forest plot indicated that there is heterogeneity among studies $\left(\mathrm{I}^{2}=99 \%, p<0.0001\right)$. The result in fixed effect suggests the need to fit the random effect model to handle heterogeneity.

In random effect model, tau ${ }^{2}$ using Dar-Simonial Liard (DL) quantified the amount of total heterogeneity to be
0.0046. Using double arcsine transformed proportion in random effect model, the weighted average from each study using the inverse of variances yield a more valid effect size.

Accordingly, the pooled perinatal mortality rate in Ethiopia was 51.3 per 1000 total births with 95\% CI $(40.8,62.8)$ (Fig. 2). Perinatal mortality includes stillbirth and early neonatal death. The pooled stillbirth rate was 36.9 per 1000 births with $95 \%$ CI $(27.3,47.8)$. The pooled early neonatal mortality rate was 29.5 per 1000 live births with 95\% CI $(23.9,35.6)$.

\section{Sensitivity analysis}

The result of sensitivity analysis indicated that no study is found to be removed since the estimate of each study, when removed, is within the confidence interval of the pooled perinatal mortality rate $(40.8,62.8)$ (Figure S1 in Additional file 4).

\section{Subgroup analysis}

The results of subgroup analysis for magnitude of perinatal mortality rate was indicated below (Table 2).

\section{Publication bias}

The presence of small study effects/publication bias was examined using regression test (Egger test). The test result indicated that there was no any small study effects or publication bias $(P=0.076)$.

\section{Trend of perinatal mortality}

The cumulative meta-analysis indicated that, there is slight reduction trend with some ups and downs in between years of publication. From the Fig. 3, we can 


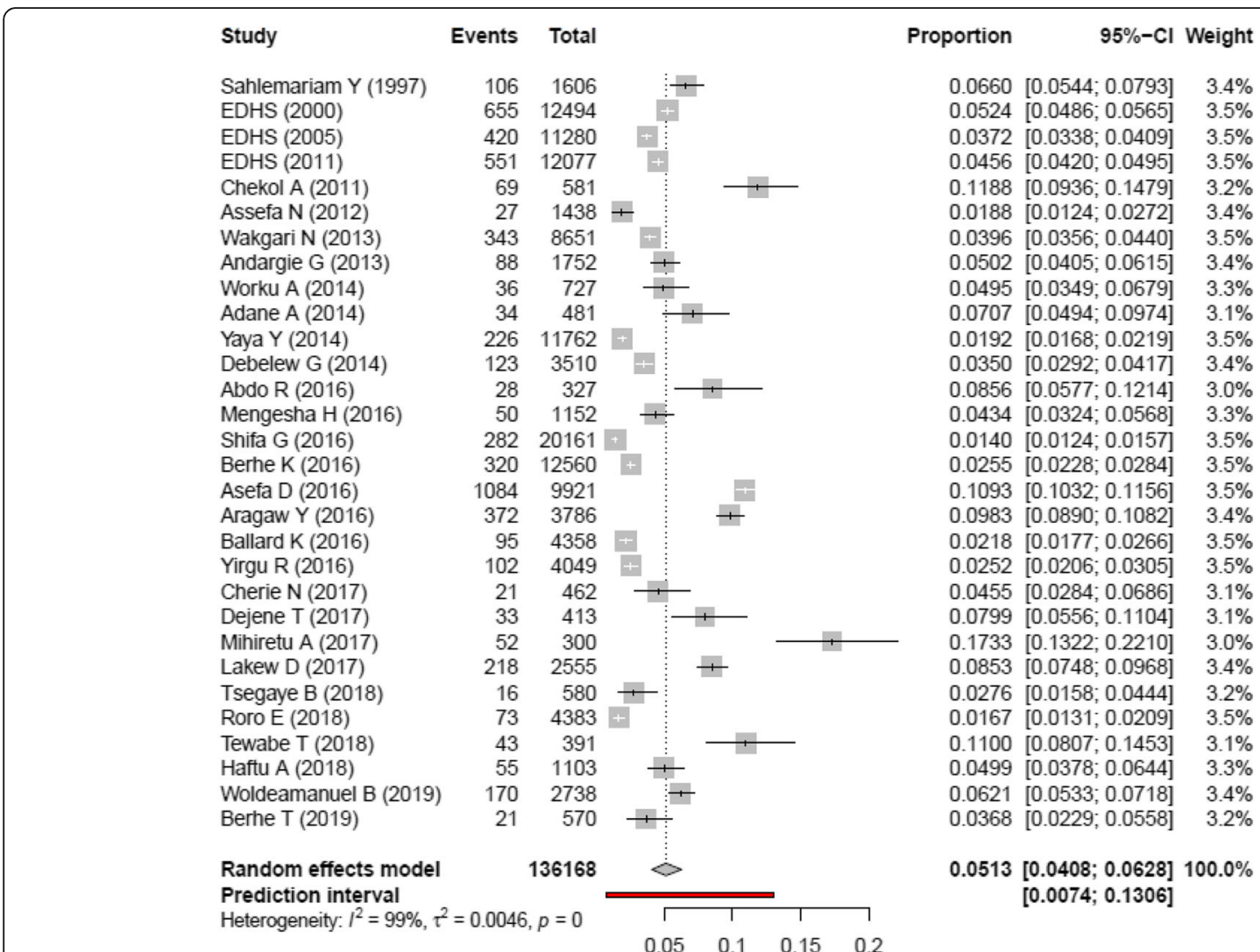

Fig. 2 Forest plot showing heterogeneity among included studies to estimate perinatal mortality rate, 1997-2019, Ethiopia. In Fig. 2: Each squared box indicates the sample size of individual studies. The horizontal line at the middle of each box indicates the $95 \%$ confidence interval of individual studies. The dot at the middle of the squared box and the horizontal line indicates the effect size (PMR) of individual studies. The long horizontal line inside the squared box which is wider than the squared box indicates the sample size is smaller than expected. The diamond shape at the end of the broken vertical line indicates the pooled effect size (pooled PMR). The red color horizontal line shows the prediction interval. The boxes at the left side of the broken vertical line show the effect sizes of individual studies that are lower than the pooled effect size and vice versa

see that; perinatal mortality rate was reduced from 66 per 1000 total births in 1997 to 51.3 per 1000 total births in 2019. Significant reduction trend in perinatal mortality rate was observed from 1997 to 2014 ( $p=$ $0.014)$ but in the last 5 years (2015-2019) no reduction was seen. Overall, regression test indicated that there was no statistically significant reduction trend in perinatal mortality rate from 1997 to $2019(P=$ $0.08)$. Since perinatal mortality rate was the sum of stillbirths and early neonatal mortality rates it is important to see the trends of them separately. In this regard, different kinds of trends were observed for stillbirth and early neonatal mortality rates (Fig. 4). There was no significant reduction trend in stillbirth rate rather it increased from 23.7 in 1997 to 36.9 in 2019 per 1000 births $(P=0.25)$. A significant reduction trend was observed in early neonatal mortality rate from 51 in 1997 to 29.5 in 2019 per 1000 live births $(P=0.01)$.

\section{Relationship between inter-pregnancy interval and} perinatal mortality

The relationship between inter-pregnancy interval less than 15 months and perinatal mortality was observed. Accordingly, children who were conceived within 15 months of their preceding child birth were nearly three times more likely risked to death during perinatal periods as compared to their counter parts; OR $=2.76,95 \%$ CI: $(2.1,3.6)$. There is low heterogeneity among studies $\left(\mathrm{I}^{2}=46 \%, P=0.07\right)$ (Fig. 5).

\section{Publication bias}

Regression test for funnel plot asymmetry showed that there is no evidence of publications bias for trim and fill analysis $(P=0.313)$ (Fig. 6).

\section{Key findings of the review}

Based on the review questions the following findings were obtained (Table 3). 
Table 2 The pooled perinatal mortality rate for subgroup analysis in random effect model, Ethiopia

\begin{tabular}{|c|c|c|c|}
\hline Variables & Characteristics & Pooled perinatal mortality rate per 1000 total births (95\% Cl) & $\mathrm{Tau}^{2}$ \\
\hline \multirow[t]{3}{*}{ Year of publication } & $1997-2000$ & $57.9(45.4-71.9)$ & 0.0003 \\
\hline & $2001-2014$ & $43.6(33.9-54.4)$ & 0.0015 \\
\hline & 2015-2019 & $54.8(36.9-76)$ & 0.0083 \\
\hline \multirow[t]{2}{*}{ Study place } & Community & $35(27-44.1)$ & 0.0022 \\
\hline & Institution & $75(58.6-93.2)$ & 0.0035 \\
\hline \multirow[t]{8}{*}{ Region } & National DHS & $44.9(36.7-53.9)$ & 0.0003 \\
\hline & Addis Ababa & $66(54.4-78.7)$ & Not applicable \\
\hline & Amhara & $59.1(37.1-85.8)$ & 0.0045 \\
\hline & Benishangual & $118.8(93.6-146.4)$ & Not applicable \\
\hline & Oromia & $53.2(22-96.8)$ & 0.0107 \\
\hline & SNNP & $46.2(28.6-67.6)$ & 0.0024 \\
\hline & Tigray & $49.1(38.6-60.8)$ & 0.0005 \\
\hline & Mixed $^{a}$ & $28.6(19.4-39.5)$ & 0.0007 \\
\hline \multirow[t]{3}{*}{ Study design } & Cross-sectional & $58(44.2-73.6)$ & 0.0051 \\
\hline & Follow up & $43.4(32.3-55.9)$ & 0.0013 \\
\hline & Nested Case Control & 20.7 (13.1-29.9) & 0.0004 \\
\hline \multirow[t]{3}{*}{ Study setting } & Urban & 45.5 (15.5-90) & 0.0039 \\
\hline & Rural & $20.5(17.4-23.8)$ & 0.0001 \\
\hline & Both & $58.6(45.7-72.8)$ & 0.0049 \\
\hline \multirow[t]{3}{*}{ Study outcome } & Stillbirth & $51.4(36.2-29)$ & 0.0035 \\
\hline & ENM & $44.4(21.6-74.8)$ & 0.0041 \\
\hline & Both(PM) & $52.7(39.3-68)$ & 0.0039 \\
\hline
\end{tabular}

Mixed $^{\text {a }}$ When the study considered more than one region

\section{Discussion}

In this review, the pooled estimate of perinatal mortality rate, stillbirth rate and early neonatal mortality rate remained high. An increasing trend was seen in stillbirth rate while decreasing trend in early neonatal mortality rate from 1997 to 2019. Insignificant reduction trend was observed in overall perinatal mortality rate. An inter-pregnancy interval of less than 15 months was found to be statistically significantly associated with perinatal mortality.

Accordingly, the pooled perinatal mortality rate in Ethiopia was 51.3 per 1000 total births (stillbirths and live births). The result was higher than the reported pooled estimate for 21 countries in four sub-regions of

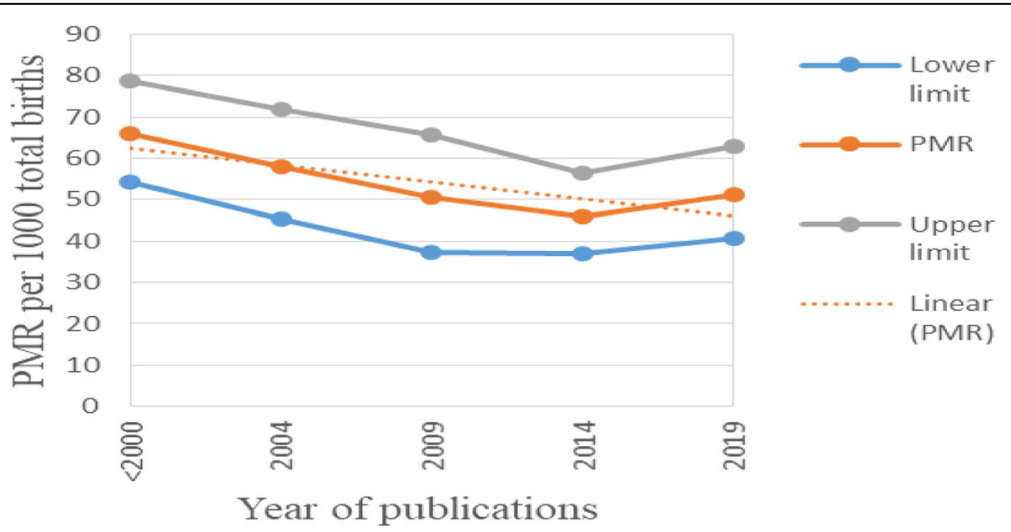

Fig. 3 Trend of perinatal mortality rate, 1997-2019, Ethiopia. In fig. 3: The diamond shaped box and respective estimates across the junctions show the trend of perinatal mortality rate (PMR) per 1,000 total births for each corresponding year of publication. 

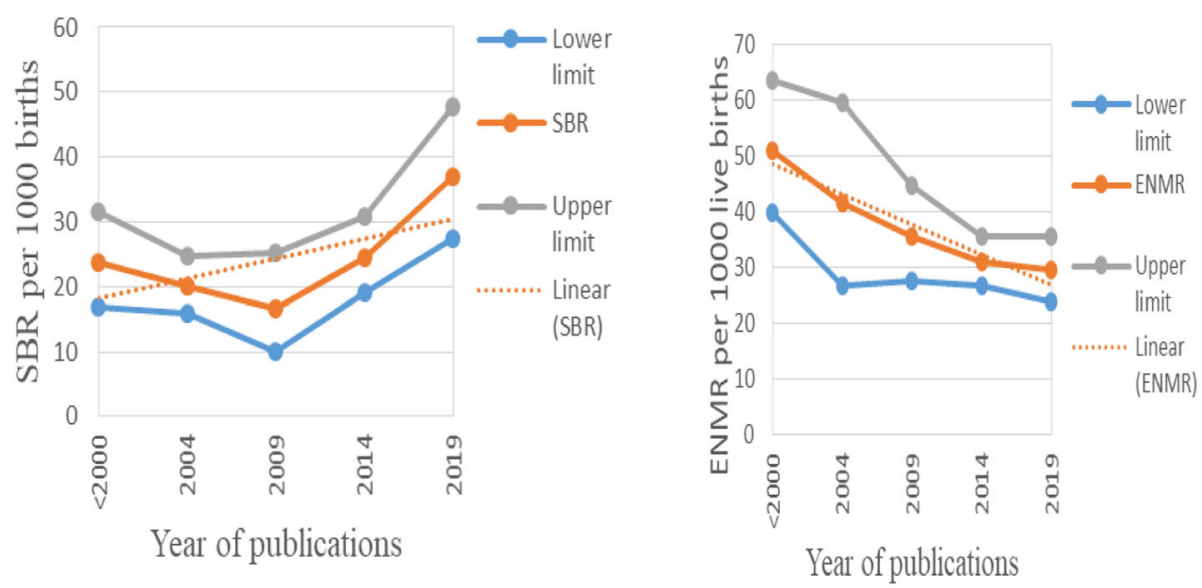

Fig. 4 Trends of stillbirth rate (SBR) and early neonatal mortality rate (ENMR), 1997-2019, Ethiopia. In Fig. 4: The diamond shaped box and respective estimates across the junctions show the trend of stillbirth rate (SBR) per 1000 births and early neonatal mortality rate (ENMR) per 1000 live births for each corresponding year of publication

Sub-Saharan Africa (SSA), 34.7 per 1000 births [48]. It is also higher than the reported pooled estimates for East African (34.5), West Africa (35.7), South Africa (30.3) and Central Africa (30.7) per 1000 births [48]. This implies that Ethiopia is a country with the highest burden of perinatal mortality in the African region. The differences might be due to the nature of studies considered for the meta-analysis. For this review, we considered published articles in addition to EDHS report. But the review in SSA considered only DHS reports for each country. The sample size and the settings considered across the regions might be the possible reasons for the difference in pooled estimates. In addition to that differences in awareness of the community, accessibility and utilization of maternal and child health services across the regions might be varied. In a country with large number of population, like Ethiopia, access to and quality of health services could be difficult. The topography and geographic location of health facilities together with lack of transportation in cases of emergencies might contribute for high perinatal death in Ethiopia. Home delivery is one of the main reasons for high prevalence of perinatal mortality because most of the complications that result in perinatal death happen during labor and delivery. Delay in seeking health facility delivery could also be the reason as most women in Ethiopia seek health care in cases of labor complication. Newborns couldn't get the medical care that they need during emergencies when they born at home and are likely to die. Even if most women visit antenatal care, they did not give birth at health facilities. Most of the time women give birth at health facilities when they had bad pregnancy outcome or complications during their previous child birth. Otherwise, home delivery is considered as a norm because when women give births at home they get various kind of social support from significant others. This social support is lacked when they visit health facility.

The trend of a perinatal mortality rate in Ethiopia indicated that there was no sharp decline rather some ups and downs seen during different times. Studies from surveillance sites in Ethiopia showed that no more changes over time regarding perinatal mortality, especially stillbirth [5]. In this review, it is also observed that the trend of stillbirth remains alarmingly increasing. However, reduction trend was observed in early neonatal mortality. Therefore, up and downs trend in perinatal mortality rate was probably due to different patterns of trends in stillbirth and early neonatal mortality rates. Decreasing trend in early neonatal mortality rate might be the reflection of due emphasis given for the reduction of neonatal mortality in order to achieve MDGs and SDGs. A specifically targeted goal was lacking for the reduction of stillbirth. Thus, stillbirth needs due emphasis of policy actions. The subgroup analysis indicated that perinatal mortality at the community and health facility levels were 35 and 75 per 1000 total births respectively. This review showed some reduction as compared to the previous systematic review done in Ethiopia that indicated no reduction in perinatal mortality over decades rather it was stagnated at 40 and 90 per 1000 total births at community and health facility respectively [49]. The difference might be due to the difference in overall sample size, inclusion criteria, time considered to include primary studies, data sources accessed for the reviews and the effect of health interventions. In overall, consistent reduction is lacking in perinatal mortality, particularly stillbirth. 


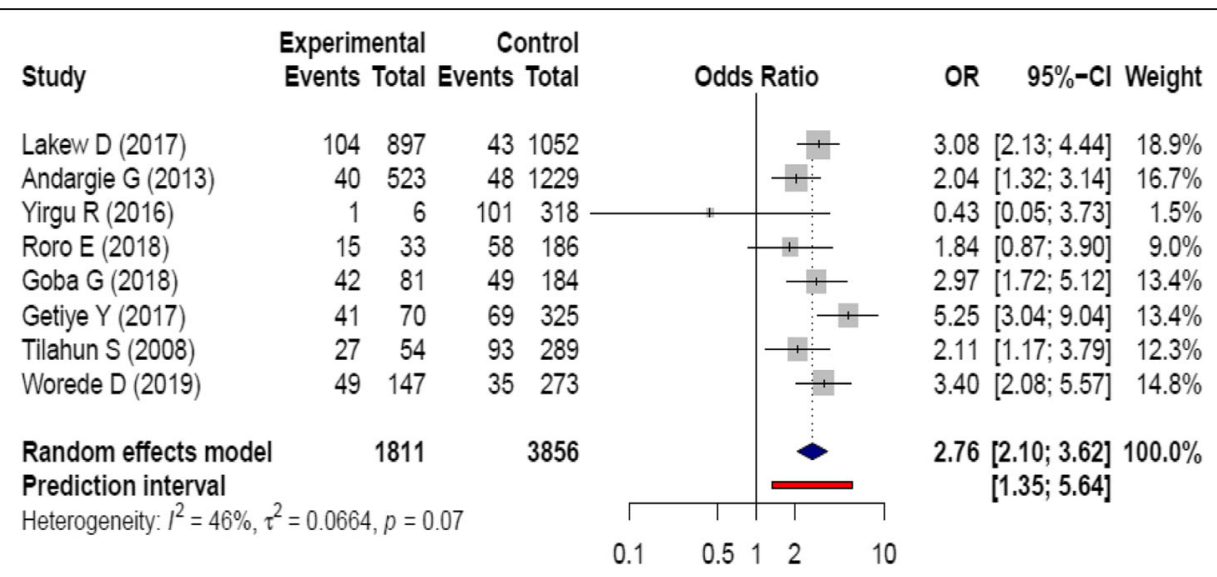

Fig. 5 Forest plot showing heterogeneity for the relationship between inter-pregnancy interval and perinatal mortality, Ethiopia. In Fig. 5: Description of the figure is similar with that of Fig. 2 with the following exceptions: the full vertical line indicates no association. A horizontal line of squared box crossing the full vertical line indicates $95 \% \mathrm{Cl}$ crossing 1 (i.e. no association)

In this review, we observed that, children who were conceived within 15 months of their preceding child birth were nearly three times more likely risked to death during the perinatal period as compared to their counter parts $[\mathrm{OR}=2.76,95 \% \mathrm{CI}$ : 2.1-3.6]. This result was supported by study conducted in Tanzania [13]. However, all primary studies included considered short interpregnancy interval less than 15 months with interpregnancy interval greater than or equal to 15 months. This categorization lacked international comparison since inter-pregnancy interval has not yet standardized. Other intervals also need to be studied to identify the optimal interval.

The National policy and decision makers need to understand an observed perinatal mortality rate in
Ethiopia is one of the highest in Africa. Unless perinatal mortality is reduced, reducing neonatal mortality might be difficult since most of the neonatal mortality occurs within the first week of life that is a part of perinatal death. Existing interventions also need to be evaluated since there was no significant reduction in perinatal mortality (mainly stillbirth) for decades. All future interventions that are aimed at improving maternal and child health status need to consider perinatal health. Due emphasis also needed to be given for modern contraceptive utilization so as to enhance perinatal health because modern contraception is the main tool that we have at hand to reduce perinatal mortality related with poorly spaced pregnancies.

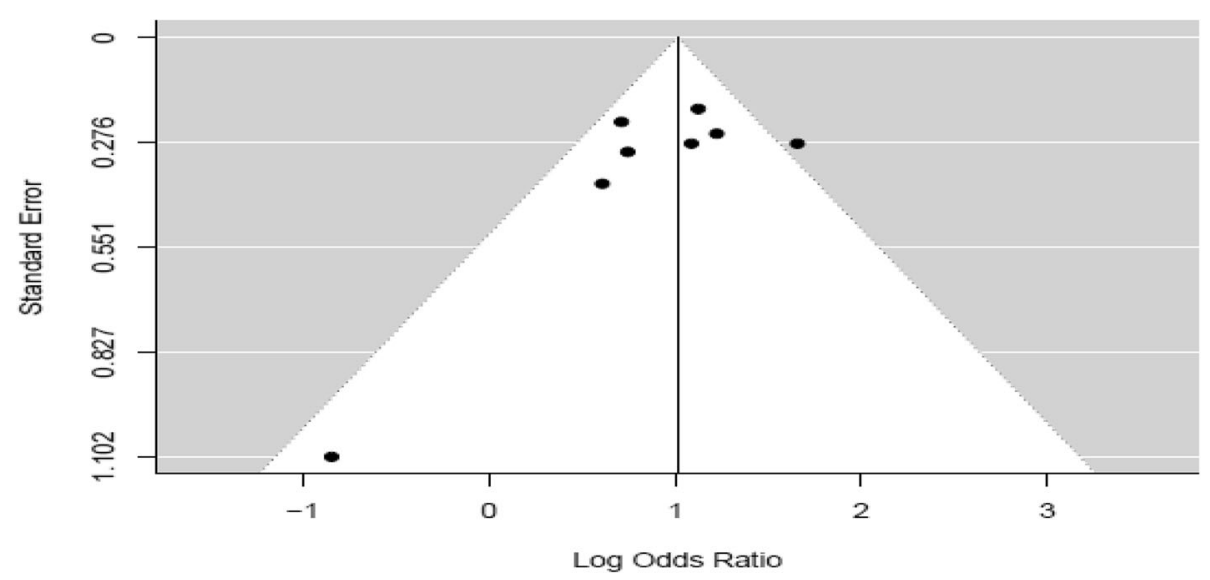

Fig. 6 Funnel plot showing publication bias for the relationship between inter-pregnancy interval and perinatal mortality, Ethiopia. In Fig. 6: Each black dot represents a single study. The $y$-axis is the standard error of the effect estimate. The x-axis is transformed effect size (log odds ratio). Larger studies (large sample size) with higher power are placed towards the top and lower studies (small sample size) with lower power are placed at the bottom. Symmetrical distribution of dots on the both sides of the vertical line inside the triangle (funnel) shows relatively no publication bias (subjective) 
Table 3 Key findings of the review

\begin{tabular}{lll}
\hline Variables & Effect sizes & $95 \% \mathrm{Cl}$ \\
\hline Perinatal mortality rate & 51.3 per 1000 total births & $40.8,62.8$ \\
Stillbirth rate & 36.9 per 1000 births & $27.3,47.8$ \\
Early neonatal mortality rate & 29.5 per live births & $23.9,35.6$
\end{tabular}

Women who have had pregnancy within 15 months of their preceding child birth were 2.76 times more likely faced perinatal mortality (stillbirths and early neonatal deaths) as compared to their counterparts (OR $2.76,95 \% \mathrm{Cl}: 2.1,3.6$ )

This review revealed that spacing pregnancies for at least 15 months was associated with reducing perinatal mortality by $64 \%$ (95\% CI: $52.38,72.38 \%)$. Further achievement can be obtained by increasing pregnancy interval for up to 24 months as recommended by World Health Organization. In the absence of randomized controlled trial that provide strong evidence, observational studies provide the valid estimate [50]. Therefore, this review could provide moderate evidence on the problem studied with Ethiopian context.

This meta-analysis may have some limitations since it is limited to observational studies published and some local sources might not be accessed. Estimated magnitude of perinatal mortality in Ethiopia may be lacked national representativeness because no data were found for regions not included in the final analysis. Time-trend analysis might not reflect the exact trend because all the years did not have reported data. Lack of controlling for confounding factors might affect the pooled effect size for the relationship between inter-pregnancy interval and perinatal mortality. Estimating inter-pregnancy interval from birth interval might result in some biases as perinatal deaths are commonly occur among preterm deliveries. Interpretations of the estimates need to consider contextual limitations. Beyond its limitation, this review will provide useful information for policy and decision makers, local planners and health workers to give due attention for prevention.

\section{Conclusions}

In this review, a perinatal mortality rate remains high in Ethiopia. Insignificant reduction trend was observed in overall perinatal mortality rate from 1997 to 2019. Stillbirth rate was alarmingly increasing while early neonatal mortality rate was promisingly decreasing. Spacing pregnancy for at least 15 months was associated with reducing perinatal mortality by $64 \%$. Counseling women on importance of spacing and strengthening modern contraceptive use will help in reducing perinatal mortality related to poor pregnancy spacing.

\section{Supplementary information}

Supplementary information accompanies this paper at https://doi.org/10. 1186/s12884-020-03089-2.

Additional file 1: Preferred Reporting Items for Systematic Review and Meta-Analysis statement (PRISMA 2009) guideline with 27 items checklist.

Additional file 2: Advanced search string.

Additional file 3: Table S1. Weaknesses of primary studies and JBI quality scores.

Additional file 4: Figure S1. Sensitivity Analysis.

\section{Abbreviations}

EDHS: Ethiopia Demographic and Health Survey; ENMR: Early neonatal mortality rate; IPI: Inter-pregnancy interval; MeSH: Medical Subjects Heading; PMR: Perinatal mortality rate; SBR: Stillbirth rate

\section{Acknowledgments}

We would like to thank all authors of primary studies included in this review.

\section{Authors' contributions}

$\mathrm{BH}, \mathrm{GA}, \mathrm{KA}$ and $\mathrm{YK}$ contributed in designing systematic review; $\mathrm{BH}, \mathrm{GA}$ and KA checked the data and conduct meta-analyses; $\mathrm{BH}, \mathrm{GA}, \mathrm{KA}$ and YK contributed in writing and editing the paper; $\mathrm{BH}, \mathrm{GA}, \mathrm{KA}$ and $\mathrm{YK}$ read and confirmed the final version of the manuscript.

Funding

There was no grant received from funding agencies.

\section{Availability of data and materials}

The datasets prepared and analyzed for this review are available in Table 1 and also from the corresponding author up on request reasonably.

Ethics approval and consent to participate

No ethical clearance is obtained as this is not a primary research.

Consent for publication

Not applicable.

Competing interests

The authors declare no competing interests exist.

\section{Author details}

${ }^{1}$ Department of Public Health, College of Medicine and Health Sciences, Wachemo University, Hossana, Ethiopia. ${ }^{2}$ Department of Health System and Policy, Institute of Public Health, College of Medicine and Health Sciences, University of Gondar, Gondar, Ethiopia. ${ }^{3}$ Department of Epidemiology and Biostatistics, Institute of Public Health, College of Medicine and Health Sciences, University of Gondar, Gondar, Ethiopia.

Received: 3 January 2020 Accepted: 3 July 2020

Published online: 29 July 2020

\section{References}

1. Goba GK, Tsegay H, Gebregergs GB, Mitiku M, Kim KA, Alemayehu M. A facility-based study of factors associated with perinatal mortality in Tigray, northern Ethiopia. Int J Gynaecol Obstet. 2018;141(1):113-9.

2. Central Statistical Agency [Ethiopia] and ICF International. Ethiopia demographic and health survey 2011. Addis Ababa and Calverton: Central Statistical Agency and ICF International; 2012.

3. Andargie G, Berhane $Y$, Worku A, Kebede $Y$. Predictors of perinatal mortality in rural population of Northwest Ethiopia: a prospective longitudinal study. BMC Public Health. 2013;13:168.

4. World Health Organization. Neonatal and perinatal mortality: country, regional and global estimates. 2006.

5. Ethiopian Universities Research Centers' Network. Trends and Causes of Perinatal Death in Ethiopia, 2010-14. Health and Demographic Surveillance system: Ethiopian Universities research centers' network policy brief number 2, 2016. 
6. United Nations Assembly General. Sustainable development goals (SDGs), transforming our world: the 2030. 2015.

7. Worku B, Kassie A, Mekasha A, Tilahun B, Worku A. Predictors of early neonatal mortality at a neonatal intensive care unit of a specialized referral teaching hospital in Ethiopia. Ethiop J Health Dev. 2012;26(3):200-7.

8. Shifa GT, Ahmed AA, Yalew AW. Early days of life are crucial for child survival in Gamo Gofa zone, Southern Ethiopia: a community based study. BMC Pediatr. 2016;16:30.

9. Central Statitical Agency [Ethiopia] and ICF International. Ethiopia demographic and health survey 2016. Addis Ababa and Calverton: Central Statistical Agency and ICF International; 2016.

10. Ouyang F. Recurrence of adverse perinatal outcomes in developing countries. Bull World Health Organ. 2013;91:357-67.

11. Roro EM, Sisay MM, Sibley LM. Determinants of perinatal mortality among cohorts of pregnant women in three districts of north Showa zone, Oromia region, Ethiopia: community based nested case control study. BMC Public Health. 2018;18(1):888

12. Yirgu R, Molla M, Sibley L, Gebremariam A. Perinatal mortality magnitude, determinants and causes in west Gojam: population-based nested casecontrol study. PLoS One. 2016;11(7):e0159390.

13. Mahande MJ, Obure J. Effect of interpregnancy interval on adverse pregnancy outcomes in northern Tanzania: a registry-based retrospective cohort study. BMC Pregnancy Childbirth. 2016;16(1):140.

14. Assefa B, Enkuselassie F, Yusuf $L$. Birth to pregnancy interval and its effect on prenatal outcome in Addis Ababa. Ethiop J Reprod Health. 2010;1(4):17.

15. Moher D, Liberati A, Tetzlaff J, Altman DG. The PRISMA Group (2009) preferred reporting items for systematic reviews and meta-analyses: the PRISMA statement. PLoS Med. 2009;6(7):e1000097.

16. JBI. Joanna Briggs Institute reviewers' manual: 2014 edition. Adelaide: JBl; 2014.

17. Higgins JP, Thompson SG. Quantifying heterogeneity in a meta-analysis. Stat Med. 2002;21(11):1539-58

18. Abdo RA, Endalemaw TB, Tesso FY. Prevalence and associated factors of adverse birth outcomes amongwomen attended maternity ward at Negest Elene Mohammed MemorialGeneral Hospital in Hosanna Town, SNNPR, Ethiopia. J Women's Health Care. 2016:5(4):324

19. Adane AA, Ayele TA, Ararsa LG, Bitew BD, Zeleke BM. Adverse birth outcomes among deliveries at Gondar University Hospital, Northwest Ethiopia. BMC Pregnancy Childbirth. 2014;14:90.

20. Aragaw YA. Perinatal mortality and associated factor in Jimma university specialized hospital, south West Ethiopia. Gynecol Obstet. 2016;6(409):21610932.

21. Asefa D, Akessa GM, Araya F, Amenu D, Girma W. Pattern of perinatal mortality among deliveries at Jimma University teaching hospital, SouthWest Ethiopia. J Women's Health. 2016;6(5):2.

22. Assefa $\mathrm{N}$, et al. The hazard of pregnancy loss and stillbirth among women in Kersa, East Ethiopia: a follow up study. Sex Reprod Healthc. 2012;3(3):107-12.

23. Ballard $\mathrm{K}$, Belete $Z$, Kinfu H, Tadesse $M$, Amin M, Atnafu $H$. The effect of prenatal and intrapartum care on the stillbirth rate among women in rural Ethiopia. Int J Gynaecol Obstet. 2016;133(2):164-7.

24. Berhe T, Gebreyesus H, Teklay H. Prevalence and determinants of stillbirth among women attended deliveries in Aksum general hospital: a facility based cross-sectional study. BMC Res Notes. 2019;12(1):368-6.

25. Berhie KA, Gebresilassie HG. Logistic regression analysis on the determinants of stillbirth in Ethiopia. Matern Health Neonatol Perinatol. 2016;2(1):10.

26. Chekol A. Birth outcome and HIV infection among labouring women in Assosa hospital, Southwest Ethiopia. Ethiop J Health Dev. 2011;25(1):10-6.

27. Cherie N, Mebratu A. Adverse birth out comes and associated factors among delivered mothers in Dessie referral hospital, North East Ethiopia. J Women's Health Reprod Med. 2017:1(1):4.

28. Debelew GT, Afework MF, Yalew AW. Determinants and causes of neonatal mortality in Jimma zone, Southwest Ethiopia: a multilevel analysis of prospective follow up study. PLoS One. 2014;9(9):e107184.

29. Getiye Y, Fantahun M. Factors associated with perinatal mortality among public health deliveries in Addis Ababa, Ethiopia, an unmatched case control study. BMC Pregnancy Childbirth. 2017;17(1):245.

30. Haftu A, Hagos H, Mehari MA. Pregnant women adherence level to antenatal care visit and its effect on perinatal outcome among mothers in Tigray public health institutions, 2017: cohort study. BMC Res Notes. 2018;11(1):872.
31. Lakew D, Tesfaye D, Mekonnen H. Determinants of stillbirth among women deliveries at Amhara region, Ethiopia. BMC Pregnancy Childbirth. 2017;17(1):375.

32. Central Statitical Agency [Ethiopia] and ICF International. Ethiopia demographic and health survey 2000. Addis Ababa and Calverton: Central Statistical Agency and ICF International; 2001.

33. Central Statitical Agency [Ethiopia] and ICF International. Ethiopia demographic and health survey 2005. Addis Ababa and Calverton: Central Statistical Agency and ICF International; 2005.

34. Mengesha HG, Wuneh AD, Lerebo WT, Tekle TH. Survival of neonates and predictors of their mortality in Tigray region, northern Ethiopia: prospective cohort study. BMC Pregnancy Childbirth. 2016;16(1):202.

35. Mihiretu A, Negash T, Elazar T. Perinatal death and associated factors in Wolaita Sodo referral hospital, southern Ethiopia: a facility based crosssectional study. Prim Health Care. 2017;7(269):2167-1079.

36. Sahle-Mariam Y, Berhane Y. Neonatal mortality among hospital delivered babies in Addis Ababa, Ethiopia. Ethiop J Health Dev. 1997:11:275-82.

37. Tewabe T, Mehariw Y, Negatie E, Yibeltal B. Neonatal mortality in the case of Felege Hiwot referral hospital, Bahir Dar, Amhara regional state, north West Ethiopia 2016: a one year retrospective chart review. Ital J Pediatr. 2018;44(1):57.

38. Tilahun D, Assefa T. Incidence and determinants of stillbirth among women who gave birth in Jimma University specialized hospital, Ethiopia. Pan Afr Med J. 2017;28:299.

39. Tilahun S, Gaym A. Past reproductive performance and its correlation with perinatal mortality in the current gestation at teaching hospitals in Addis Ababa, Ethiopia. Ethiop Med J. 2008:46(4):313-24.

40. Tsegaye B, Kassa A. Prevalence of adverse birth outcome and associated factors among women who delivered in Hawassa town governmental health institutions, South Ethiopia, in 2017. Reprod Health. 2018;15(1):193.

41. Wakgari N, Wencheko E. Risk factors of neonatal mortality in Ethiopia. Ethiop J Health Dev. 2013;27(3):192-9.

42. Woldeamanuel BT, Gelebo KK. Statistical analysis of socioeconomic and demographic correlates of perinatal mortality in Tigray region, Ethiopia: a cross sectional study. BMC Public Health. 2019:19(1):1-10.

43. Worede DT, Dagnew GW. Determinants of stillbirth in Felege-Hiwot comprehensive specialized referral hospital, north-west, Ethiopia, 2019. BMC Res Notes. 2019;12(1):1-6.

44. Worku AG, Yalew AW, Afework MF. The contributions of maternity care to reducing adverse pregnancy outcomes: a cohort study in Dabat district, Northwest Ethiopia. Matern Child Health J. 2014;18(6):1336-44.

45. Yaya Y, Eide KT, Norheim OF, Lindtjørn B. Maternal and neonatal mortality in south-West Ethiopia: estimates and socio-economic inequality. PLoS One. 2014;9(4):e96294.

46. Landis JR, Koch GG. The measurement of observer agreement for categorical data. Biometrics. 1977;33(1):159-74.

47. Gizaw M, Molla M, Mekonnen W. Trends and risk factors for neonatal mortality in Butajira District, south Central Ethiopia, (1987-2008): a prospective cohort study. BMC Pregnancy Childbirth. 2014;14:64.

48. Akombi BJ, Renzaho AM. Perinatal mortality in Sub-Saharan Africa: a metaanalysis of demographic and health surveys. Ann Global Health. 2019;85(1): 106.

49. Berhan Y, Berhan A. Perinatal mortality trends in Ethiopia. Ethiop J Health Sci. 2014;24:29-40.

50. Concato J, Shah N, Horwitz RI. Randomized, controlled trials, observational studies, and the hierarchy of research designs. N Engl J Med. 2000;342(25): 1887-92.

\section{Publisher's Note}

Springer Nature remains neutral with regard to jurisdictional claims in published maps and institutional affiliations. 